PHYSICAL REVIEW E 94, 069903(E) (2016)

\title{
Publisher's Note: Laser propagation in dense magnetized plasma [Phys. Rev. E 94, 053207 (2016)]
}

S. X. Luan, W. Yu, F. Y. Li, Dong Wu, Z. M. Sheng, M. Y. Yu, and J. Zhang

(Received 29 November 2016; published 6 December 2016)

DOI: 10.1103/PhysRevE.94.069903

This paper was published online on 14 November 2016 with an incorrect ordering of affiliations. The author list and affiliations should read as S. X. Luan, ${ }^{1}$ W. Yu, ${ }^{1}$ F. Y. Li, ${ }^{2}$ Dong Wu, ${ }^{1}$ Z. M. Sheng, ${ }^{2,3}$ M. Y. Yu, ${ }^{4,5}$ and J. Zhang ${ }^{3}$

${ }^{1}$ State Key Laboratory of High Field Laser Physics, Shanghai Institute of Optics and Fine Mechanics, Chinese Academy of Science, Shanghai 201800, China

${ }^{2}$ SUPA, Department of Physics, University of Strathclyde, Glasgow G4 0NG, United Kingdom

${ }^{3}$ Laboratory for Laser Plasmas and Department of Physics and Astronomy, Shanghai Jiao Tong University, Shanghai 200240, China

${ }^{4}$ Institute of Fusion Theory and Simulation, Zhejiang University, Hangzhou 310027, China

${ }^{5}$ Institute for Theoretical Physics I, Ruhr University, D-44780 Bochum, Germany

The affiliations have been corrected as of 29 November 2016. The affiliations are correct in the printed version of the journal. 